\title{
Acute appendicitis caused by endometriosis: a case report
}

\author{
Styliani Laskou', Theodossis S Papavramidis ${ }^{1 *}$, Angeliki Cheva² ${ }^{1}$ Nick Michalopoulos ${ }^{1}$, Charilaos Koulouris ${ }^{1}$, \\ Isaak Kesisoglou ${ }^{1}$ and Spiros Papavramidis ${ }^{1}$
}

\begin{abstract}
Introduction: Endometriosis is a well-recognized gynecological condition in the reproductive age group. Surgical texts present the gynecological aspects of the disease in detail, but the published literature on unexpected manifestations, such as appendiceal disease, is inadequate. The presentation to general surgeons may be atypical and pose diagnostic difficulty. Thus, a definitive diagnosis is likely to be established only by the histological examination of a specimen.
\end{abstract}

Case presentation: We report a case of endometriosis of the appendix in a 25-year-old Caucasian woman who presented with symptoms of acute appendicitis and was treated by appendectomy, which resulted in a good outcome.

Conclusions: We discuss special aspects of acute appendicitis caused by endometriosis to elucidate the pathologic entity of this variant of acute appendicitis.

\section{Background}

Endometriosis is the presence of endometrial glands and stroma outside the uterine cavity and musculature [1]. It affects $4 \%$ to $50 \%$ of women of reproductive age and results in pelvic pain in up to $50 \%$ of these patients [2]. The symptomatology of the disease is often related to the location of the lesions [3], and for that reason endometriosis of the gastrointestinal tract, although rare, may cause a wide spectrum of symptoms [4-6]. Appendiceal endometriosis not only may cause symptoms of acute appendicitis [7-10] but also is known to cause cyclic and chronic right lower quadrant pain [11], melena [12], lower intestinal hemorrhage [13], cecal intussusceptions $[14,15]$ and intestinal perforation, especially during pregnancy [16].

Appendiceal endometriosis was first described in 1860 [17], while in 1951 Collins [12] reviewed a total of 150 cases in the literature. Four years afterward Collins further described more than 50,000 random pathologic assessments of the appendix and reported the prevalence of appendiceal endometriosis as $0.054 \%$ [18]. More

\footnotetext{
* Correspondence: papavramidis@hotmail.com

'Third Department of Surgery, AHEPA University Hospital, Aristotle University of Thessaloniki, Thessaloniki, Greece Full list of author information is available at the end of the article
}

recent studies, however, have reported the prevalence of appendiceal endometriosis to be around 0.8\% [19].

We describe a case of a woman with appendiceal endometriosis that presented as acute appendicitis. We additionally discuss special aspects of the disease to elucidate this variant of acute appendicitis.

\section{Case presentation}

A 25-year-old Caucasian woman was admitted to our hospital with a two-day history of lower quadrant abdominal pain. She had no fever, but she reported nausea, vomiting and anorexia. Her McBurney's point was positive with abdominal guarding and rigidity. She had no relevant gynaecological history.

The patient's white blood cell count was $12,400 / \mathrm{mm}^{3}$ with $83 \%$ neutrophils. Her urine analysis was normal, and her urine pregnancy test was negative. Acute appendicitis was diagnosed, and an appendectomy was performed. Intraoperatively, the appendix appeared mildly congested. The appendix measured $6.5 \times 0.6 \mathrm{~cm}$ at the widest diameter. The pathological examination revealed small nodules found in the wall of the appendix. The patient's ectopic endometrial glands were surrounded by endometrial stroma (Figure 1). The pathology report led to the diagnosis of appendiceal endometriosis. Postoperatively,
Ciomed Central 


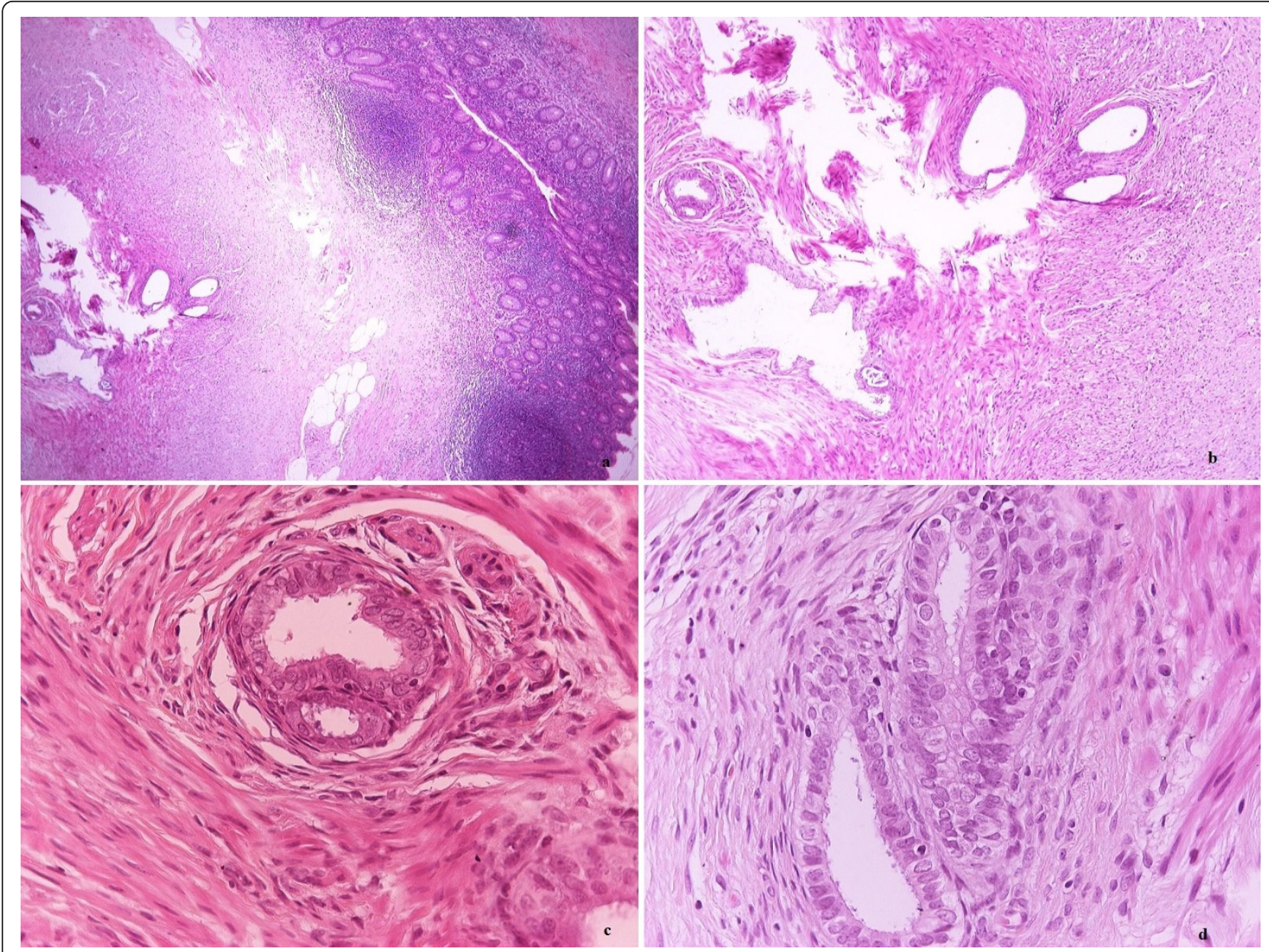

Figure 1 Small nodules found in the wall of the appendix. The endometrial glands are surrounded by endometrial stroma.

the patient recovered with no residual pain. Today, five years after the patient's appendectomy, her gynecologic anamnestic record remains clear and her follow-up with echograms has revealed no other sites of endometriosis.

\section{Discussion}

When endometrial tissue is found outside its normal location, it is called "endometriosis". This condition is seen in $10 \%$ of women within their menstrual age. It is called "adenomyosis" or "internal endometriosis" when the endometrial tissue is found within the uterine muscles. External endometriosis is commonly found in the genital organs and the pelvic peritoneum [20].

The true prevalence of extragenital endometriosis is unknown because of a lack of large, well-defined case series. Case reports throughout the literature describe extragenital endometriosis in almost every organ and tissue in the body [21]. It may be seen in the gastrointestinal system, omentum, mesentery, liver, operation scars and, rarely, in the kidneys, lungs, central nervous system, skin and extremities [20-22]. Interestingly, one of the only sites where extragenital endometriosis has not been reported is the spleen [21]. With regard to the type of appendiceal endometriosis that we describe here, its incidence is thought to be low and is considered to be between $0.054 \%$ and $0.8 \%[18,19]$.

Several theories have been proposed to explain the pathogenesis of extragenital endometriosis [22]. The implantation or retrograde menstruation theory proposes that endometrial tissue from the uterus is transported in a retrograde fashion through the fallopian tubes [23]. The direct transplantation theory and the dissemination theory can explain extrapelvic endometriosis $[24,25]$. The coelomic metaplasia theory hypothesizes that the peritoneal cavity contains progenitor cells or cells capable of differentiating into endometrial tissue $[26,27]$. The induction theory suggests that sloughed endometrium produces substances to form endometriosis. The embryonic rest theory claims that a specific stimulus to a Müllerian origin cell nest produces endometriosis. The most recently developed theory is the cellular immunity theory, which suggests that 
alterations in cell-mediated and humoral immunity allow ectopic endometrial cells to proliferate [22].

Appendiceal endometriosis patients can be categorized into four groups in terms of symptomatology: (1) patients who present with acute appendicitis; (2) patients who present with appendix invagination; (3) patients manifesting atypical symptoms such as abdominal colic, nausea and melena; and (4) patients who are asymptomatic. These four patient groups are discussed in the subsections that follow.

Acute appendiceal inflammation can arise because of partial or complete luminal occlusion by the endometrioma [28]. Another mechanism suggested is that of endometrium hemorrhage within the seromuscular layer of appendix, which is followed by edema, obstruction and inflammation. Pain in the right lower abdominal quadrant is one of the most common symptoms, and one-third of those patients present with a typical appendiceal symptomatology [20]. The routine examination of a patient suspected of having acute appendicitis consists of a complete blood count and urine analysis. The most important diagnostic tool is still a physical examination, but use of imaging studies is increasing day-by-day. This is a result of the need for early diagnosis and treatment to achieve a lower perforation rate and fewer complications [29]. Leukocytosis with the predominance of polymorphonuclear leukocytes accompanies acute appendicitis in most cases, along with elevated C-reactive protein. In our patient, fever was absent, but there was an increase in leukocytes. Computed tomographic scans obtained to diagnose appendiceal endometriosis often show a distended, nonopacified appendix without inflammation [30].

Along with foreign bodies, inflammation, polyps and neoplasia, endometriosis should be considered as a possible cause of appendiceal invagination [20]. Appendiceal intussusception is uncommon (incidence of $0.01 \%$ ). Endometrial involvement of the appendix is usually accompanied by chronic fibrosis, inflammation and hyperplasia or hypertrophy of the muscularis propria. This hypertrophic segment serves as a lead point for hyperperistalsis, hence making it prone to invagination, particularly when combined with a fully mobile appendix that has a wide proximal lumen and a fat-free mesoappendix. Patients often present with weeks to months of intermittent abdominal pain, nausea, vomiting, melena (or "currant jelly stool"), fever or constipation [31]. Occasionally, patients are asymptomatic. The radiographic findings are generally normal unless a small-bowel obstruction exists. Sonography may identify the classic target lesion, or "donut sign," associated with intussusception [32]. Computed tomographic abdominal scans may demonstrate a soft tissue mass in the region of the cecum, although it may not lead to the diagnosis [28].
Patients who fall within these groups do not manifest signs of either appendicitis or ileus. These two groups are usually diagnosed incidentally [20].

Appendiceal endometriosis is often seen in patients with ovarian endometriosis. Appendectomies were performed in 65 of 125 patients with ovarian endometriosis who underwent various operations because of infertility. Thirteen of the appendectomy pathological examinations revealed appendiceal endometriosis. This result has led to a discussion whether to perform elective appendectomies in patients who have undergone gynecological operations because of endometriosis [20]. Moreover, endometriosis of the appendix is reported to have a high incidence of association with leiomyoma of the uterus and menstrual abnormalities [8]. Some authors have even reported the cases of endometriosis patients with symptoms of abdominal pain with menstruation. However, our patient had no history of these abnormalities, and her symptoms did not coincide with menstruation.

Appendiceal endometriosis is diagnosed pathologically. Glandular tissue, endometrial stroma and hemorrhage are typical examinations conducted in patients with endometriosis [20]. About half of endometriosis of the appendix involves the body and half involves the tip of the appendix. Muscular and seromuscular involvement occurs in two-thirds of patients, while the serosal surface is involved in only one-third of patients. The mucosa is not involved, but Langman et al. [33] found that the submucosa was involved in one-third of patients with endometriosis of the appendix. In their series, the endometriotic foci were also found in the muscle, serosa and subserosa. There was no correlation between the location of the endometriotic foci and the patients' symptoms [33]. Therefore, mucosal or submucosal endometriosis is much more likely to mimic primary inflammatory diseases such as Crohn's disease, infectious or ischemic enteritis or colitis, or mucosal prolapse than endometriosis of the outer bowel wall [31]. Our patient is categorized in the typical form of appendiceal endometriosis, since small nodules were present in the wall of the appendix while the endometrial glands were surrounded by endometrial stroma.

The treatment consists mainly of surgery and hormone therapy. The treatment tends to be determined by the age of the patient and the degree of the patient's symptoms. Thus, the extent of resection should be appropriate. Intraoperative investigations usually result in an accurate diagnosis of endometriosis with minimal resection. A gynecological assessment should be performed to determine the extent of endometriosis, and postoperative follow-up is mandatory for appendiceal endometriosis. In our patient, the postoperative gynecological examination did not reveal any 
other endometriotic lesions [30]. Laparoscopic appendectomy is now commonly performed for appendicitis. Laparoscopic surgery is useful for women with chronic abdominal pain caused by endometriosis, ovarian cysts, adhesions and hernias. Laparoscopy enables the exploration of the total peritoneal cavity and the selection of the appropriate method for a definitive diagnosis. Medical treatments for endometriosis are secondary. Appendiceal endometriosis appears to be an incidental finding and one that is not clinically important [31].

\section{Conclusion}

Appendiceal endometriosis is rare, and its preoperative diagnosis is difficult. However, it should be included in the differential diagnosis of acute abdominal pain, especially when women of childbearing age present with clinical symptoms of acute appendicitis but no evidence is observed on imaging studies. Laparoscopy is useful for the diagnosis, and appendectomy relieves the acute symptoms.

\section{Consent}

Written informed consent was obtained from the patient for publication of this case report and accompanying images. A copy of the written consent is available for review by the Editor-in-Chief of this journal.

\section{Author details}

'Third Department of Surgery, AHEPA University Hospital, Aristotle University of Thessaloniki, Thessaloniki, Greece. ${ }^{2}$ Department of Pathology, AHEPA University Hospital, Aristotle University of Thessaloniki, Thessaloniki, Greece.

\section{Authors' contributions}

SL analyzed and interpreted the patient data and drafted the manuscript. TSP received the patient in the outpatient department. NM and CK received the patient in the outpatient department, served as auxiliary surgeons and drafted the manuscript. AC performed the pathological examination and was a major contributor in writing the manuscript. IK was the principal surgeon and drafted the manuscript. SP was responsible for the overall treatment of the patient and corrected the manuscript. All authors read and approved the final manuscript.

\section{Competing interests}

The authors declare that they have no competing interests.

Received: 24 August 2010 Accepted: 11 April 2011 Published: 11 April 2011

\section{References}

1. Olive DL, Schwartz LB: Endometriosis. N Engl J Med 1993, 328:1759-1769.

2. Cramer DW, Missmer SA: The epidemiology of endometriosis. Ann N Y Acad Sci 2002, 955:11-22, discussion 34-36, 396-406.

3. Vercellini P, Trespidi L, De Giorgi O, Cortesi I, Parazzini F, Crosignani PG: Endometriosis and pelvic pain: relation to disease stage and localization. Fertil Steril 1996, 65:299-304.

4. Kratzer GL, Salvati EP: Collective review of endometriosis of the colon. Am J Surg 1955, 90:866-869.

5. Macafee $\mathrm{CH}$, Greer HL: Intestinal endometriosis: a report of 29 cases and a survey of the literature. J Obstet Gynaecol Br Emp 1960, 67:539-555.
6. Thierstein ST, Allen E: A comparative analysis of the diagnosis and treatment of endometriosis, including a report of 53 cases of intestinal endometriosis. Am J Obstet Gynecol 1946, 51:635.

7. Thiel CW: Endometriosis of the appendix and cecum associated with acute appendicitis. Minn Med 1986, 69:20-21.

8. Khoo JJ, Ismail MS, Tiu CC: Endometriosis of the appendix presenting as acute appendicitis. Singapore Med J 2004, 45:435-436.

9. Mittal VK, Choudhury SP, Cortez JA: Endometriosis of the appendix presenting as acute appendicitis. Am J Surg 1981, 142:519-522.

10. Stefanidis K, Kontostolis S, Pappa L, Kontostolis E: Endometriosis of the appendix with symptoms of acute appendicitis in pregnancy. Obstet Gynecol 1999, 93:850.

11. Harris RS, Foster WG, Surrey MW, Agarwal SK: Appendiceal disease in women with endometriosis and right lower quadrant pain. J Am Assoc Gynecol Laparosc 2001, 8:536-541.

12. Collins DC: Endometriosis of the vermiform appendix: review of literature, with addition of nine new instances, one of which caused severe melena. AMA Arch Surg 1951, 63:617-622.

13. Shome GP, Nagaraju M, Munis A, Wiese D: Appendiceal endometriosis presenting as massive lower intestinal hemorrhage. Am J Gastroenterol 1995, 90:1881-1883.

14. Panzer S, Pitt HA, Wallach EE, Thuluvath PJ: Intussusception of the appendix due to endometriosis. Am J Gastroenterol 1995, 90:1892-1893.

15. Sakaguchi N, Ito M, Sano K, Baba T, Koyama M, Hotchi M: Intussusception of the appendix: a report of three cases with different clinical and pathologic features. Pathol Int 1995, 45:757-761.

16. Nakatani $Y$, Hara M, Misugi K, Korehisa H: Appendiceal endometriosis in pregnancy. Report of a case with perforation and review of the literature. Acta Pathol Jpn 1987, 37:1685-1690.

17. Von Rokitansky C: Ueber Uterusdrusen-Neubildung in Uterus- und Ovarial-Sarcomen. Ztschr kk Gesselsh Aerzte Wien 1860, 16:577.

18. Collins DC: A study of 50,000 specimens of the human vermiform appendix. Surg Gynecol Obstet 1955, 101:437-445.

19. Berker B, Lashay N, Davarpanah R, Marziali M, Nezhat CH, Nezhat C: Laparoscopic appendectomy in patients with endometriosis. $J$ Minim Invasive Gynecol 2005, 12:206-209.

20. Uncu H, Taner D: Appendiceal endometriosis: two case reports. Arch Gynecol Obstet 2008, 278:273-275.

21. Apostolidis S, Michalopoulos A, Papavramidis TS, Papadopoulos VN, Paramythiotis D, Harlaftis N: Inguinal endometriosis: three cases and literature review. South Med J 2009, 102:206-207.

22. Papavramidis TS, Sapalidis K, Michalopoulos N, Karayanopoulou G, Raptou G, Tzioufa V, Kesisoglou I, Papavramidis ST: Spontaneous abdominal wall endometriosis: a case report. Acta Chir Belg 2009, 109:778-781.

23. Seli $E$, Berkkanoglu M, Arici A: Pathogenesis of endometriosis. Obstet Gynaecol Clin North Am 2003, 30:41-61.

24. Ichimiya M, Hirota T, Muto M: Intra-lymphatic embolic cells with cutaneous endometriosis in the umbilicus. J Dermatol 1998, 25:333-336.

25. Halban J: Metastatic hystero-adenosis. Wien Klin Wochenschr 1924, 37:1205-1206.

26. Steck WD, Helwig EB: Cutaneous endometriosis. Clin Obstet Gynaecol 1966, 9:373-383

27. Matsuura $\mathrm{K}$, Ohtake $\mathrm{H}$, Katabuchi $\mathrm{H}$, Okamura $\mathrm{H}$ : Coelomic metaplasia theory of endometriosis: evidence from in vivo studies and an in vitro experimental model. Gynaecol Obstet Invest 1999, 27:18-20.

28. ljaz S, Lidder S, Mohamid W, Carter M, Thompson H: Intussusception of the appendix secondary to endometriosis: a case report. J Med Case Reports 2008, 2:12

29. Memisoglu K, Karip B, Mestan M, Onur E: The value of preoperative diagnostic tests in acute appendicitis, retrospective analysis of 196 patients. World J Emerg Surg 2010, 5:5

30. Douglas C, Rotimi O: Extragenital endometriosis: a clinicopathological review of a Glasgow hospital experience with case illustrations. J Obstet Gynaecol 2004, 24:804-808.

31. Idetsu A, Ojima H, Saito K, Yamauchi H, Yamaki E, Hosouchi Y, Nishida Y, Kuwano H: Laparoscopic appendectomy for appendiceal endometriosis presenting as acute appendicitis: report of a case. Surg Today 2007, 37:510-513.

32. Luzier J, Verhey $P$, Dobos N: Preoperative $C T$ diagnosis of appendiceal intussusception. AJR Am J Roentgenol 2006, 187:W325-W326. 
33. Langman J, Rowland R, Vernon-Roberts B: Endometriosis of the appendix. Br J Surg 1981, 68:121-124.

doi:10.1186/1752-1947-5-144

Cite this article as: Laskou et al:: Acute appendicitis caused by

endometriosis: a case report. Journal of Medical Case Reports 2011 5:144

Submit your next manuscript to BioMed Central and take full advantage of:

- Convenient online submission

- Thorough peer review

- No space constraints or color figure charges

- Immediate publication on acceptance

- Inclusion in PubMed, CAS, Scopus and Google Scholar

- Research which is freely available for redistribution

Submit your manuscript at 